\title{
Martingales and arbitrage: a new look
}

\section{Alejandro Balbás and Pedro Jiménez-Guerra}

\begin{abstract}
This paper addresses the equivalence between the absence of arbitrage and the existence of equivalent martingale measures. The equivalence will be established under quite weak assumptions since there are no conditions on the set of trading dates (it may be finite or countable, with bounded or unbounded horizon, etc.) or on the trajectories of the price process (for instance, they do not have to be right-continuous).

Besides we will deal with arbitrage portfolios rather than free-lunches. The concept of arbitrage is much more intuitive than the concept of free lunch and has more clear economic interpretation. Furthermore it is more easily tested in theoretical models or practical applications.

In order to overcome the usual mathematical difficulties arising when dealing with arbitrage strategies, the set of states of nature will be widened by drawing on projective systems of Radon probability measures, whose projective limit will be the martingale measure. The existence of densities between the "real" probabilities and the "risk-neutral" probabilities will be guaranteed by introducing the concept of "projective equivalence". Hence some classical counter-examples will be solved and a complete characterization of the absence of arbitrage will be provided in a very general framework.
\end{abstract}

\section{Martingalas y arbitraje: un nuevo enfoque}

Resumen. Analizaremos la equivalencia entre la ausencia de arbitraje y la existencia de una medida de martingala. Esta equivalencia se establecerá bajo supuestos débiles, puesto que no hay condiciones sobre el conjunto de fechas de negociación (puede ser finito o contable, con horizonte acotado o no acotado, etc.) ni sobre las trayectorias del proceso de precios (por ejemplo, no tienen que ser continuas por la derecha).

Trabajaremos con el concepto de arbitraje, y no con el de free-lunch. La noción de arbitraje es mucho más intuitiva y tiene una interpretación económica mucho más clara, además de ser más fácil de verificar en las aplicaciones prácticas.

Para salvar dificultades matemáticas, extenderemos el conjunto de estados de la naturaleza mediante el uso de sistemas proyectivos de probabilidades regulares (de Radon), cuyo límite proyectivo será la medida de martingala. La existencia de densidades entre las "probabilidades reales" y las "neutrales al riesgo" se garantizará mediante la introducción del concepto de "equivalencia proyectiva". Algunos contra-ejemplos clásicos serán resueltos, y una caracterización completa de la ausencia de arbitraje será presentada en un contexto muy general.

\section{Introduction}

The existence of pricing rules, discount factors or state prices is crucial in the literature on capital markets. It is closely related to the concepts of arbitrage and equilibrium (see, for instance, Chamberlain and Roth-

Palabras clave / Keywords: Arbitrage, martingale measure, projective system.

Mathematics Subject Classifications: 91B28, 91B70. 
schild [8, (1983)] or Hansen and Jagannathan [15, (1997)]). Harrison and Kreps [16, (1979)] showed the link between pricing rules and martingale measures.

Since Harrison and Kreps [16, (1979)] established the existence of martingale probability measures for some arbitrage free pricing models their result has been extended in multiple directions, generating the Fundamental Theorem of Asset Pricing (henceforth FTAP). For instance, Dalang et al. [11, (1990)], Schachermayer [22, (1992)] and [23, (1994)], Delbaen and Schachermayer [13, (1998)] or Jacod and Shiryaev [17, (1998)] provide deep characterizations of the existence of martingale measures in different settings.

Nevertheless a simple version of the FTAP cannot be proved, in the sense that the arbitrage absence is not sufficient to build martingale measures if the set of trading dates is not finite. It was pointed out in Back and Pliska [2, (1991)], where a simple counter-example is provided. To overcome this problem Clark [10, (1993)] introduced the concept of "free lunch", far weaker than the concept of arbitrage. The absence of free lunch has been the key to yield further extensions of the FTAP, even in the imperfect market case (see for instance Jouini and Kallal [18, (1995)]).

Any free lunch can be understood as an "approximated arbitrage" in the sense that it is "quite close" to an arbitrage portfolio. However, it is almost an arbitrage but it is not an arbitrage, it is not so intuitive and its economic interpretation is not so clear. On the contrary it is introduced in mathematical terms and solves a mathematical problem, but classical pricing models (binomial model, Black and Scholes model, etc.) usually deal with the concept of arbitrage.

Besides, if possible, it may be worth to provide risk-neutral probabilities and pricing rules (martingale measures) under simple and meaningful assumptions, as the arbitrage absence. This is in the line of many others Representation Theorems of Mathematical Finance. For instance, the representation of coherent risk measures (Artzner et al. [1, (1999)]) or pricing rules in one period imperfect markets (Chateauneuf et al. [9, (1996)], De Waegenaere et al. [12, (2003)], Castagnoli et al. [7, (2004)] etc.) is addressed by using intuitive hypotheses.

Balbás et al. [5, (2002)] have shown that it is possible to characterize the arbitrage absence if the set of trading dates is $\mathbb{N}$, the set of natural numbers. They built an appropriate countable projective system $\left(\nu_{n}\right)_{n \in \mathbb{N}}$ of perfect probability measures (see Musial [21, (1980)]) that are risk-neutral for each finite subset $\{0,1, \ldots, n\} \subset \mathbb{N}$. Then they showed that the projective limit $\nu$ is risk-neutral for the whole set of trading dates $(\mathbb{N})$, in the sense that the set of states of the world and the price process may be extended to a "new price process" which is a martingale under $\nu$. The initial probability measure $\mu$ and $\nu$ cannot be equivalent, as illustrated by using the counter example of Back and Pliska. However, for any finite subset of $\mathbb{N}$, the projections of $\mu$ and $\nu$ are equivalent, and there are Radon-Nikodyn derivatives in both directions. Balbás et al. used this property to introduce the concept of "projective equivalence" of probability measures.

The interest of the approach above seems to be clear, since it even permits us to extend the classical FTAP for infinitely many trading dates without using the projective equivalence, i.e., in the classical setting. For instance, Balbás et al. [3, (2007)] have used the analysis of Balbás et al. [5, (2002)] so as to extend the FTAP in a model with dynamically bounded Sharpe ratios. Unbounded Sharpe ratios would lead to very high returns with bounded risk level, which is barely acceptable in Financial Economics. The findings of Balbás et al. [3, (2007)] have some relationships with those of Föllmer and Schachermayer [14, (2008)], where the authors show that the absence of martingale measures in the long run provokes the existence of asymptotic arbitrage that is related to the market price of risk.

This paper follows the approach of Balbás et al. [5, (2002)] and extends the analysis bearing in mind a much wider scope. Even usual constraints, also imposed in the literature when dealing with free lunches, are no assumed here. For instance, there are no conditions on the set of trading dates (it may be finite or countable, with bounded or unbounded horizon, etc.) or on the trajectories of the price process (for example, they do not have to be right-continuous).

The existence of risk-neutral probabilities will be stated by means of projective limits of projective systems of Radon probability measures (see Schwartz [24, (1973)]), rather than projective systems of perfect measures. These projective systems will permit us to broaden the set of states of nature and to generalize the concept of projective equivalence. 
The outline of the article is as follows. Section 2 will introduce the basic concepts and notations. Section 3 will summarize some mathematical background that will be often applied. Section 4 will transform the problem in order to introduce the "projective price process". Section 5 will be devoted to provide martingale measures when the set of trading dates is countable. The most important result is Theorem 5 and generalizes those findings of Balbás et al. [5, (2002)], since the set $\mathcal{T}$ of trading dates does not have to be similar to $\mathbb{N}$. For example, $\mathcal{T}$ can equal the set of non-negative rational numbers or, more generally, $\mathcal{T}$ can have adherent points, and every adherent point may belong to $\mathcal{T}$ or to its complementary. It seems to be a significant extension since, for instance, CadLag price processes are characterized for their values at rational dates. Section 6 will conclude the article.

\section{Preliminaries and notations}

Let $(\Omega, \mathcal{F}, \mu)$ be a probability space composed of the set $\Omega$, the $\sigma$-algebra $\mathcal{F}$ and the probability measure $\mu$. Suppose that $\mathcal{T} \subset[0, \infty)$ is a set (finite or infinite, with finite or infinite horizon) of trading dates such that $0 \in \mathcal{T}$ ( 0 denoting the current date) and $\mathcal{T}$ contains at least two elements. As usual, the arrival of information will be provided by the increasing family $\left[\mathcal{F}_{t}\right]_{t \in \mathcal{T}}$ of $\sigma$-algebras of $\Omega$ such that $\mathcal{F}_{0}=\{\emptyset, \Omega\}$ and $\sigma\left(\bigcup_{t \in \mathcal{T}} \mathcal{F}_{t}\right)=\mathcal{F}, \sigma\left(\bigcup_{t \in \mathcal{T}} \mathcal{F}_{t}\right)$ being the $\sigma$-algebra generated by the algebra $\bigcup_{t \in \mathcal{T}} \mathcal{F}_{t}$. The restriction of $\mu$ to $\mathcal{F}_{t}$ will be denoted by $\mu_{t}$ for every $t \in \mathcal{T}$.

Consider $n$ different securities whose prices will be represented by the $\mathbb{R}^{n}$-valued adapted stochastic process

$$
\{S(\omega, t): \omega \in \Omega, t \in \mathcal{T}\}
$$

Obviously

$$
S(\omega, t)=\left(S_{1}(\omega, t), S_{2}(\omega, t), \ldots, S_{n}(\omega, t)\right)
$$

where $S_{j}(\omega, t) \in \mathbb{R}$ for $\omega \in \Omega, t \in \mathcal{T}$ and $j=1,2, \ldots, n$, and represents the price of the $j$-th asset at $t$ under the state $\omega$. In order to simplify the notation the previous processes may be also denoted by $S$ and $S_{j}$, $j=1,2, \ldots, n$. Analogous notations and conventions will be used for any other $\mathbb{R}$-valued or $\mathbb{R}^{n}$-valued adapted stochastic process.

As usual, the first asset will play the role of a numeraire, and therefore we will impose that

$$
S_{1}(\omega, t)=1
$$

holds for every $\omega \in \Omega$ and $t \in \mathcal{T}$.

For a fixed $\omega \in \Omega$ the corresponding path or trajectory of $S$ will be denoted by $S(\omega,-)$, while for any fixed trading date $t \in \mathcal{T}$ the symbol $S(-, t)$ yields the random variable providing us with prices at $t$. Similar notations will be used in similar situations.

Consider arbitrary an finite subsets $\left\{t_{0}=0<t_{1}<\cdots<t_{k}\right\} \subset \mathcal{T}$. For such a subset consider any stochastic process

$$
x: \Omega \times\left\{0<t_{1}<\cdots<t_{k}\right\} \longrightarrow \mathbb{R}^{n}
$$

adapted to the filtration $\left[\mathcal{F}_{t}\right]_{t \in\left\{0<t_{1}<\cdots<t_{k}\right\}}$. Then, $x$ is said to be a self-financing portfolio if

$$
\left[x\left(\omega, t_{i}\right)-x\left(\omega, t_{i-1}\right)\right] S\left(\omega, t_{i}\right)=0
$$

$\mu$-a.s. and $i=1,2, \ldots, k{ }^{1}$ The set of self-financing portfolios will be denoted by $\mathcal{A}$.

Let $x \in \mathcal{A}$. It may be easily proved that the above set of trading dates may be extended by adding a finite number of elements of $\mathcal{T}$ and the convention $x\left(-, t_{i}\right)-x\left(-, t_{i-1}\right)=0$ if $t_{i}$ is a new element that does not belong to the initial finite set of trading dates. Therefore it is easy to show that $\alpha_{1} x^{1}+\alpha_{2} x^{2} \in \mathcal{A}$ if $x^{i} \in \mathcal{A}$ and $\alpha_{i} \in \mathbb{R}, i=1,2$.

\footnotetext{
${ }^{1}$ Notice that products in (2) are scalar (or inner) products of $\mathbb{R}^{n}$.
} 
If $x \in \mathcal{A}$ then

$$
\lambda(x)=x(\omega, 0) S(\omega, 0) \in \mathbb{R}
$$

will be its current price, while the $\mathcal{F}_{t_{k}}$-measurable random variable

$$
x\left(\omega, t_{k}\right) S\left(\omega, t_{k}\right)
$$

will be its final pay-off and will be denoted by $\Lambda(x)$, or $\Lambda(x)(\omega)$ if necessary.

As usual, an arbitrage portfolio allows traders to obtain "money without risk".

Definition 1 A self-financing portfolio $x \in \mathcal{A}$ is said to be an arbitrage if

a) $\lambda(x) \leq 0$

b) $\Lambda(x)(\omega) \geq 0, \quad \mu$-a.s.

c) $\mu(\omega \in \Omega: \Lambda(x)(\omega)-\lambda(x)>0)>0$.

Hereafter we will assume essentially bounded prices, i.e., $S_{j}(-, t) \in L^{\infty}\left(\mathcal{F}_{t}\right)$ for $j=1,2, \ldots, n$ and $t \in \mathcal{T}$. Thus for every $t \in \mathcal{T}$ there exists a $\mu$-null set $Z_{t} \in \mathcal{F}_{t}$ such that the inequality

$$
\|S(\omega, t)\| \leq\|S(-, t)\|_{\infty}
$$

holds for every $\omega \in \Omega \backslash Z_{t}$.

Given $t, s \in \mathcal{T}, t \leq s$, it makes sense to introduce the conditional expectation of $S(-, s)$ with respect to $\mathcal{F}_{t}$ and will be denoted by $E_{\mu}\left(S(-, s) \mid \mathcal{F}_{t}\right)$. Similar notations will be used for similar cases.

Definition 2 Given a finite set $\left\{0<t_{1}<\cdots<t_{k}\right\} \subset \mathcal{T}$ and a probability measure $\nu: \mathcal{F}_{t_{k}} \mapsto[0,1]$ then $\nu$ is said to be a martingale measure on $\left\{t_{0}=0<t_{1}<\cdots<t_{k}\right\}$ if $\mu_{t_{k}}$ and $\nu$ are equivalent and

$$
E_{\nu}\left(S\left(-, t_{i}\right) \mid \mathcal{F}_{t_{j}}\right)=S\left(-, t_{j}\right)
$$

whenever $i, j=0,1, \ldots, k$ and $i \geq j$.

The absence of arbitrage and the FTAP guarantee the existence of martingale measures on any finite set of trading dates (see for instance Dalang et al. [11, (1990)], Schachermayer [22, (1992)] or Jacod and Shiryaev [17, (1998)]).

Theorem 1 The model is arbitrage free if and only if there exists a martingale measure on every finite set $\left\{0<t_{1}<\cdots<t_{k}\right\} \subset \mathcal{T}$.

Despite the previous result, the counter-example of Back and Pliska [2, (1991)] points out that the martingale measure depends on $\left\{0<t_{1}<\cdots<t_{k}\right\}$, i.e., in general, it is not possible to find $\nu: \mathcal{F} \mapsto[0,1]$ equivalent to $\mu$ and such that (4) holds for every $t_{i}, t_{j} \in \mathcal{T}$ with $t_{i} \geq t_{j}$.

\section{Some mathematical background}

It is worth to recall some properties concerning Radon measures and projective systems of topological spaces and measures. Further details may be found in Kelley [19, (1955)], Bourbaki [6, (1969)], Schwartz [24, (1973)] or Musial [21, (1980)].

If $X$ is a Hausdorff topological space and $\mathcal{B}$ represents its Borel $\sigma$-algebra then a finite and positive measure $\nu$ on $\mathcal{B}$ is said to be a Radon measure if

$$
\nu(B)=\sup \{\nu(K): K \subset B, K \text { compact }\}
$$


for every $B \in \mathcal{B}$. It is easy to show that (5) implies

$$
\nu(B)=\inf \{\nu(G): G \supset B, G \text { open }\}
$$

for every $B \in \mathcal{B}$. Furthermore (5) leads to the existence of an open subset $G$ of $X$ such that $\nu(G)=0$ and $G \supset G^{\prime}$ for every $G^{\prime}$ open and such that $\nu\left(G^{\prime}\right)=0$. Its complementary $X \backslash G$ is a closed set called the support of $\nu$ and denoted by $\operatorname{Sp}(\nu)$.

If $X$ is compact then the set $\mathcal{R}$ of positive and finite Radon measures on $\mathcal{B}$ may be identified with the set of $\mathbb{R}$-valued, linear, continuous and positive functionals on $\mathcal{C}(X)(\mathcal{C}(X)$ being the Banach space of $\mathbb{R}$-valued and continuous functions on $X$ ), and the Alaoglu's Theorem trivially leads to the weak ${ }^{*}$-compactness of $\mathcal{R}_{0}=\{\nu \in \mathcal{R}: \nu(X)=1\}$.

The Hausdorff topological space $X$ is said to be a Radon space if every finite and positive measure on $\mathcal{B}$ is a Radon measure. Every Polish space (metric, complete and separable) is a Radon space. In particular, $\mathbb{R}^{C}$ is a Radon space for every countable set $C, \mathbb{R}^{C}$ being the space of $\mathbb{R}$-valued functions on $C$ endowed with the simple (or product) topology.

For any $(\mathcal{F}, \mathcal{B})$-measurable function $f: \Omega \rightarrow X$, the measure $\mu$ induces a measure on $\mathcal{B}$, called the image measure $f(\mu)$, given by $f(\mu)(A)=\mu\left(f^{-1}(A)\right)$ for all $A \in \mathcal{B}$. $^{2}$ Obviously $f(\mu)$ is Radon if so is the space $X$.

If $X$ and $Y$ are two compact and Hausdorff spaces and $f: X \rightarrow Y$ is a continuous function then $f$ induces the new function $\mathcal{R}^{X} \ni \nu \rightarrow f(\nu) \in \mathcal{R}^{Y}, \mathcal{R}^{X}$ and $\mathcal{R}^{Y}$ being the sets of finite and positive Radon measures on $X$ and $Y$ respectively. It is easy to show that this transformation is continuous if $\mathcal{R}^{X}$ and $\mathcal{R}^{Y}$ are endowed with their respective weak $^{*}$-topologies.

Let $\leq$ be the ordering relation of a directed set $I$. Consider a family of Hausdorff topological spaces $\left(X_{i}\right)_{i \in I}$ and the continuous maps $\pi_{i j}: X_{j} \rightarrow X_{i}, i, j \in I, i \leq j$. We say that $\left(\left(X_{i}, \pi_{i j}\right) ; i, j \in I, i \leq j\right)$ is a projective system of topological spaces if $\pi_{i k}=\pi_{i j} \circ \pi_{j k}$ for all $i, j, k \in I, i \leq j \leq k$. Its projective limit is

$$
X=\left\{\left(x_{i}\right)_{i \in I} \in \prod_{i \in I} X_{i}: x_{j}=\pi_{j k}\left(x_{k}\right) \text { if } j, k \in I, j \leq k\right\}
$$

endowed with the product topology. Clearly, the canonical projections $\pi_{i}: X \rightarrow X_{i}, i \in I$, are continuous and satisfy $\pi_{i}=\pi_{i j} \circ \pi_{j}$ for all $i, j \in I, i \leq j$. X may be endowed with its Borel $\sigma$-algebra $\mathcal{B}$ and the cylindrical $\sigma$-algebra $\mathcal{B}_{0} \subset \mathcal{B}$ generated by

$$
\bigcup_{i \in I}\left\{\pi_{i}^{-1}\left(A_{i}\right): A_{i} \subset X_{i}, A_{i} \text { open }\right\}
$$

Note that $\pi_{i}$ is $\mathcal{B}_{0}$-measurable for every $i \in I$.

Under the notations above, if $\nu_{i}$ is a Radon measure on $X_{i}$ for every $i \in I$ and $\pi_{i j}\left(\nu_{j}\right)=\nu_{i}$ if $i, j \in I$, $i \leq j$, then $\left(\nu_{i}\right)_{i \in I}$ is said to be a projective system of Radon measures. We will say that $\left(\nu_{i}\right)_{i \in I}$ converges to the measure $\nu: \mathcal{B}_{0} \rightarrow \mathbb{R}$ (or $\nu$ is the projective limit of $\left(\nu_{i}\right)_{i \in I}$ ) if $\pi_{i}(\nu)=\nu_{i}$ for every $i \in I$.

The following results on the existence of projective limits of projective systems of Radon measures are adapted from Bourbaki [6, (1969)] and Schwartz [24, (1973)] respectively.

Theorem 2 Under the notations above, if $I=\mathbb{N}$ then every projective system of Radon measures $\left(\nu_{i}\right)_{i \in \mathbb{N}}$ has a projective limit $\nu$. Moreover, $\nu$ is unique and can be extended to a unique Radon measure defined over the Borel $\sigma$-algebra $\mathcal{B}$.

Theorem 3 (Prokhorov) Under the notations above there exists a Radon measure $\nu: \mathcal{B} \rightarrow \mathbb{R}$ such that $\pi_{i}(\nu)=\nu_{i}$ for every $i \in I$ if and only if for every $\varepsilon>0$ there exists a compact set $K \subset X$ with $\nu_{i}\left(X_{i} \backslash \pi_{i}(K)\right)<\varepsilon$ for every $i \in I$. In the affirmative case $\nu$ is unique.

\footnotetext{
${ }^{2}$ Notice that $(\Omega, \mathcal{F}, \mu)$ may be replaced by any other measure space.
} 
Corollary 1 Under the notations above if $\operatorname{Sp}\left(\nu_{i}\right) \subset X_{i}$ is compact for every $i \in I$ then there exists a unique Radon measure $\nu: \mathcal{B} \rightarrow \mathbb{R}$ such that $\pi_{i}(\nu)=\nu_{i}$ for every $i \in I$. Furthermore $\nu$ has a compact support and $\operatorname{Sp}(\nu) \subset \prod_{i \in I} \operatorname{Sp}\left(\nu_{i}\right)$.

There is a special type of projective system of Radon measures closely related to stochastic processes. So, consider $I=\mathcal{P}_{F}(\mathcal{T})$ the set of finite subsets of $\mathcal{T}$ containing $\{0\}$ and consider the order of $I$ generated by the usual inclusion. For every $V \in \mathcal{P}_{F}(\mathcal{T})$ we will take the (Polish and therefore Radon) topological space $\left(\mathbb{R}^{n}\right)^{V}$ of $\mathbb{R}^{n}$-valued functions defined on $V$. Of course, $\left(\mathbb{R}^{n}\right)^{V}$ is endowed with the product topology. If $V, U \in \mathcal{P}_{F}(\mathcal{T})$ satisfy $V \subset U$ then $\pi_{V U}:\left(\mathbb{R}^{n}\right)^{U} \rightarrow\left(\mathbb{R}^{n}\right)^{V}$ is the standard projection. It is trivial to prove that we are facing a projective system

$$
\left(\left(\left(\mathbb{R}^{n}\right)^{V}, \pi_{V U}\right): V, U \in \mathcal{P}_{F}(\mathcal{T}), V \subset U\right)
$$

of topological spaces whose projective limit can be identified with the space $\left(\mathbb{R}^{n}\right)^{\mathcal{T}}$ endowed the simple topology. Furthermore $\pi_{V}:\left(\mathbb{R}^{n}\right)^{\mathcal{T}} \rightarrow\left(\mathbb{R}^{n}\right)^{V}$ is also the standard projection for every $V \in \mathcal{P}_{F}(\mathcal{T})$.

As in the general case, the projective limit $\left(\mathbb{R}^{n}\right)^{\mathcal{T}}$ may be endowed with its Borel or cylindrical $\sigma$ algebras, denoted by $(\mathcal{B})^{\mathcal{T}}$ and $\left(\mathcal{B}_{0}\right)^{\mathcal{T}}$ respectively. When endowed with $\left(\mathcal{B}_{0}\right)^{\mathcal{T}}$ (the lower one) a function $f: \Omega \rightarrow\left(\mathbb{R}^{n}\right)^{\mathcal{T}}$ is measurable if and only if $\pi_{V} \circ f: \Omega \rightarrow\left(\mathbb{R}^{n}\right)^{V}$ is measurable for every $V \in \mathcal{P}_{F}(\mathcal{T})$ (Footnote 2 also applies here).

Suppose that $\left(\nu_{V}\right)_{V \in \mathcal{P}_{F}(\mathcal{T})}$ is a projective system of Radon measures associated with the above projective system of topological spaces. The following result may be established by readapting some statements of Kopp [20, (1984)].

Theorem 4 (Daniell-Kolmogorov) Under the notations above the system of Radon measures

$$
\left(\nu_{V}\right)_{V \in \mathcal{P}_{F}(\mathcal{T})}
$$

has a unique projective limit $\nu_{\mathcal{T}}:\left(\mathcal{B}_{0}\right)^{\mathcal{T}} \rightarrow \mathbb{R}$

\section{Projective system approach}

As in the previous section, consider $I=\mathcal{P}_{F}(\mathcal{T})$. Let $V \in \mathcal{P}_{F}(\mathcal{T})$ and let $S_{V}$ be function connecting any state of nature $\omega \in \Omega$ and the restriction on $V$ of the corresponding trajectory of the price process $S$, i.e., the function

$$
\Omega \ni \omega \longmapsto S_{V}(\omega) \in\left(\mathbb{R}^{n}\right)^{V}
$$

such that $S_{V}(\omega)(t)=S(\omega, t)$ for $\omega \in \Omega$ and $t \in V$. If $v$ is the maximum of $V$, then $S_{V}$ is $\mathcal{F}_{v}$-measurable and therefore $S_{V}\left(\mu_{v}\right)$ is a Radon probability measure on $\left(\mathbb{R}^{n}\right)^{V}$. In order to simplify the notation the previous probability measure will be represented by $S_{V}(\mu)$ and $v$ will be omitted. Now it is straightforward to prove that

$$
\left(S_{V}(\mu)\right)_{V \in \mathcal{P}_{F}(\mathcal{T})}
$$

is a projective system of Radon probability measures associated with (6). Notice that the support of $S_{V}(\mu)$ is compact for every $V \in \mathcal{P}_{F}(\mathcal{T})$ since it is included in (see (3))

$$
\operatorname{Sp}\left(S_{V}(\mu)\right) \subset \prod_{t \in V} K_{t}
$$

where for every $t \in \mathcal{T}$ we have that $K_{t}$ represents the compact ball of $\mathbb{R}^{n}$ with center at zero and radius equal to $\|S(-, t)\|_{\infty}$.

Consider now the function

$$
\Omega \ni \omega \longmapsto S_{\mathcal{T}}(\omega)=S(\omega,-) \in\left(\mathbb{R}^{n}\right)^{\mathcal{T}}
$$


$S(\omega,-)$ being the whole path associated with $\omega$. It is easy to show that the previous function is $\mathcal{F}$ measurable if $\left(\mathbb{R}^{n}\right)^{\mathcal{T}}$ is endowed with the cylindrical $\sigma$-algebra $\left(\mathcal{B}_{0}\right)^{\mathcal{T}}$ since, according to the properties of Section 3, it is equivalent to show that $\pi_{V} \circ S_{\mathcal{T}}=S_{V}$ is $\mathcal{F}$-measurable for every $V \in \mathcal{P}_{F}(\mathcal{T})$. Consequently it makes sense to consider its image measure $S_{\mathcal{T}}(\mu)$ defined on $\left(\mathcal{B}_{0}\right)^{\mathcal{T}}$ and it is straightforward to show that the probability measure $S_{\mathcal{T}}(\mu)$ is the projective limit of (7), i.e.,

$$
\pi_{V}\left(S_{\mathcal{T}}(\mu)\right)=S_{V}(\mu)
$$

for every $V \in \mathcal{P}_{F}(\mathcal{T})$. Moreover Corollary 1 and (8) prove that $S_{\mathcal{T}}(\mu)$, projective limit of $\left(S_{V}(\mu)\right)_{V \in \mathcal{P}_{F}(\mathcal{T})}$, may be extended to a unique Radon measure defined on $(\mathcal{B})^{\mathcal{T}}$. If there is no confusion then both $S_{\mathcal{T}}(\mu)$ and its extension will be denoted with the same symbol. Besides (8) leads to

$$
\operatorname{Sp}\left(S_{\mathcal{T}}(\mu)\right) \subset \prod_{t \in \mathcal{T}} K_{t} .
$$
define

Next let us introduce the projective price process. So, for every $\varpi \in\left(\mathbb{R}^{n}\right)^{\mathcal{T}}$ and every $t \in \mathcal{T}$ we can

$$
S^{*}(\varpi, t)=\varpi(t) .
$$

One can consider the filtration $\left[\mathcal{F}_{t}^{*}\right]_{t \in \mathcal{T}}$ on $\left(\mathbb{R}^{n}\right)^{\mathcal{T}}$ such that $\mathcal{F}_{t}^{*}$ is the smallest $\sigma$-algebra for which $S^{*}\left(-, t^{\prime}\right)$ is $\mathcal{F}_{t}^{*}$-measurable if $t^{\prime} \in \mathcal{T}$ and $t^{\prime} \leq t$. By construction $\left[\mathcal{F}_{t}^{*}\right]_{t \in \mathcal{T}}$ is increasing and all of them are included in the cylindrical $\sigma$-algebra $\left(\mathcal{B}_{0}\right)^{\mathcal{T}}$ because the random variable $S^{*}(-, t)$ is clearly $\left(\mathcal{B}_{0}\right)^{\mathcal{T}}$-measurable for every $t \in \mathcal{T}$.

Our major objective is to establish the existence of a probability measure $\nu:\left(\mathcal{B}_{0}\right)^{\mathcal{T}} \rightarrow[0,1]$ so that the projective price process $S^{*}$ can be a martingale with respect to $\nu$, solving this way the drawback pointed out after Theorem 1. Besides $\nu$ and $S_{\mathcal{T}}(\mu)$ should satisfy "some kind of equivalence", since the existence of Radon-Nikodyn densities seems to be desirable. We will show that the complete equivalence between both probability measures does not necessarily hold in general but we can introduce a weaker concept. Thus following the approach of Balbás et al. [5, (2002)], or Balbás and Downarowicz [4, (2007)] for a one period model with infinitely many securities, we have:

Definition 3 Two arbitrary probability measures $\nu:\left(\mathcal{B}_{0}\right)^{\mathcal{T}} \rightarrow[0,1]$ and $\xi:\left(\mathcal{B}_{0}\right)^{\mathcal{T}} \rightarrow[0,1]$ are said to be projectively equivalent if $\pi_{V}(\nu)$ and $\pi_{V}(\xi)$ are equivalent for every $V \in \mathcal{P}_{F}(\mathcal{T})$.

Obviously, if $\nu$ and $\xi$ are equivalent then they are projectively equivalent too. It will be shown that the converse does not hold in general (see also Balbás et al. [5, (2002)]).

In order to establish the existence of projectively equivalent martingale measures it is convenient to translate the arbitrage absence in terms of the projective price process $S^{*}$. So we present a result whose proof is very simple and therefore omitted.

Proposition 1 If the initial price process $S$ is arbitrage free then the projective price process $S^{*}$ is arbitrage free.

The projective price process has been built without imposing special assumptions on the set of trading dates $\mathcal{T}$. In particular, $\mathcal{T}$ might be finite. Consequently, the procedure also applies when considering any $V \in \mathcal{P}_{F}(\mathcal{T})$ instead of $\mathcal{T}$. In such a case, if $t \in V$ then $\mathcal{F}_{t}^{V *}$ denotes the smallest $\sigma$-algebra of $\left(\mathbb{R}^{n}\right)^{V}$ allowing the natural sections (the projective price process) $\left(\mathbb{R}^{n}\right)^{V} \ni \varpi \rightarrow S^{V *}\left(\varpi, t^{\prime}\right)=\varpi\left(t^{\prime}\right) \in \mathbb{R}^{n}$ to be $\mathcal{F}_{t}^{V *}$-measurable for every $t^{\prime} \in V, t^{\prime} \leq t$. It is clear that $\left(\mathcal{F}_{t}^{V *}\right)_{t \in V}$ is a increasing filtration.

Next we will translate Theorem 3 into the projective system setting. Once again we omit the proof.

Proposition 2 Let $V=\left\{t_{0}=0<t_{1}<\cdots<t_{k}\right\} \in \mathcal{P}_{F}(\mathcal{T})$ and let $\nu$ be a martingale measure in the sense of Definition 2. Then $\nu_{V}^{*}=S_{V}(\nu)$ and $S_{V}(\mu)$ are equivalent and

$$
E_{\nu_{V}^{*}}\left(S^{V *}\left(-, t_{i}\right) \mid \mathcal{F}_{t_{j}}^{V *}\right)=S^{V *}\left(-, t_{j}\right),
$$

$i, j=0,1, \ldots, k, i \geq j$. Moreover $\nu_{V}^{*}$ has a compact support included in $\prod_{t \in V} K_{t}$. 
As a consequence of the latter proposition if we were able to build a projective system by "correctly connecting $\nu_{V}^{*}$ as $V$ grows" then the projective limit, whose existence is guaranteed by Corollary 1, could be an adequate candidate to be our "risk-neutral martingale measure". The construction of this projective system will be the major goal of Section 5 .

\section{Countable sets of trading dates}

Throughout this section we will assume that $\mathcal{T}$ is countable. Thus there exists a bijection $\Phi: \mathbb{N} \leftrightarrow \mathcal{T}$ such that $\Phi(0)=0$. Denote $t_{m}=\Phi(m)$ for every $m \in \mathbb{N}$. Then the equality

$$
\mathbb{N}=\bigcup_{m=1}^{\infty}\{0,1, \ldots, m\}
$$

leads to

$$
\mathcal{T}=\Phi(\mathbb{N})=\bigcup_{m=1}^{\infty} \Phi(\{0,1, \ldots, m\})=\bigcup_{m=1}^{\infty} \mathcal{T}_{m},
$$

where $\mathcal{T}_{m}$ denotes the finite set $\left\{0, t_{1}, \ldots, t_{m}\right\}$. Hence the following result has been stated:

Lemma 1 There exists an increasing sequence $\left(\mathcal{T}_{m}\right)_{m \in \mathbb{N}}$ of finite subsets of $\mathcal{T}$ such that

$$
\mathcal{T}=\bigcup_{m \in \mathbb{N}} \mathcal{T}_{m}
$$

and $0 \in \mathcal{T}_{m}$ for every $m \in \mathbb{N}$. Furthermore, $\left(\mathbb{R}^{n}\right)^{\mathcal{T}}$ can be identified with the projective limit of the projective system of topological spaces

$$
\left(\left(\left(\mathbb{R}^{n}\right)^{\mathcal{T}_{r}}, \pi_{r, s}\right): r, s \in \mathbb{N}, r \leq s\right)
$$

where $\pi_{r, s}$ denotes the standard projection of $\left(\mathbb{R}^{n}\right)^{\mathcal{T}_{s}}$ onto $\left(\mathbb{R}^{n}\right)^{\mathcal{T}_{r}}$.

The sequence of (12) and the system (13) will be fixed throughout this section. To simplify the notation $\left(\mathbb{R}^{n}\right)^{\mathcal{T}_{r}}$ will be represented by $\left(\mathbb{R}^{n}\right)_{r}$ for every $r \in \mathbb{N}$.

Suppose that the initial model is arbitrage free and fix $r \in \mathbb{N}$. Proposition 2 ensures the existence of Radon probability measures $\nu_{r}^{*}$ on any $\left(\mathbb{R}^{n}\right)_{r}$ equivalent to $\pi_{V}\left(S_{\mathcal{T}}(\mu)\right)$ and with support included in $\prod_{t \in \mathcal{T}_{r}} K_{t}$, such that the restriction to $\left(\mathbb{R}^{n}\right)_{r}$ of the projective price process is a martingale with respect to the filtration $\left(\mathcal{F}_{t}^{r *}\right)_{t \in \mathcal{T}_{r}}{ }^{3}$ Consider the set $\mathcal{M}_{r r}$ of Radon probability measures satisfying these properties. Now if $s \geq r$ consider $\mathcal{M}_{r s}=\pi_{r s}\left(\mathcal{M}_{s s}\right)=\left\{\pi_{r s}\left(\nu_{s}^{*}\right): \nu_{s}^{*} \in \mathcal{M}_{s s}\right\}$ and it is easily proved that $\mathcal{M}_{r r} \supset \mathcal{M}_{r s}$. Define $\mathcal{M}_{r}=\bigcap_{s=r}^{\infty} \mathcal{M}_{r s} \subset \mathcal{M}_{r r}$.

Lemma 2 The sets $\mathcal{M}_{r r}$ and $\mathcal{M}_{r}$ are non void and weak*-compact for every $r \in \mathbb{N}$.

PROOF. Proposition 2 guarantees that $\mathcal{M}_{s s}$ is non void for every $s \geq r$. Moreover, every probability measure of $\mathcal{M}_{s s}$ has a compact support included in $\prod_{t \in \mathcal{T}_{s}} K_{t}$ so they can be considered Radon measures on this compact space. It is easy to see that $\mathcal{M}_{s s}$ is weak ${ }^{*}$-closed. Then the results of Section 3 show that $\mathcal{M}_{s s}$ is weak ${ }^{*}$-compact and the weak*-continuity of $\mathcal{M}_{s s} \ni \nu_{s}^{*} \rightarrow \pi_{r s}\left(\nu_{s}^{*}\right) \in \mathcal{M}_{r r}$ (see Section 3) shows that every $\mathcal{M}_{r s}$ is weak ${ }^{*}$-compact. Thus it is sufficient to show that every finite subset of $\left\{\mathcal{M}_{r s}: s \geq r\right\}$ has non void intersection. But given any finite subset $\left\{\mathcal{M}_{r s}: s=s_{1}>s_{2}>\cdots>s_{k} \geq r\right\}$ one can take $s_{0} \geq s_{1}$ and we have that $\emptyset \neq \pi_{r s_{0}}\left(\mathcal{M}_{s_{0} s_{0}}\right)=\pi_{r s_{j}} \pi_{s_{j} s_{0}}\left(\mathcal{M}_{s_{0} s_{0}}\right) \subset \pi_{r s_{j}}\left(\mathcal{M}_{s_{j} s_{j}}\right)$ for $j=1,2, \ldots, k$.

\footnotetext{
${ }^{3}$ Notice that the notation has been simplified and $\mathcal{F}_{t}^{r *}$ represents $\mathcal{F}_{t}^{\mathcal{T}_{r} *}$. Analogously, $S_{t}^{r *}$ will represent $S_{t}^{\mathcal{T}_{r} *}$.
} 
Theorem 5 Suppose that $\mathcal{T}$ is countable. If the initial model is arbitrage-free then there exists a Radon probability measure $\nu^{*}$ on $\left(\mathbb{R}^{n}\right)^{\mathcal{T}}$ such that $\operatorname{Sp}\left(\nu^{*}\right) \subset \prod_{t \in \mathcal{T}} K_{t}, \nu^{*}$ and $S_{\mathcal{T}}(\mu)$ are projectively equivalent and the projective price process $S^{*}$ is a martingale with respect to $\nu^{*}$ and the increasing filtration $\left[\mathcal{F}_{t}^{*}\right]_{t \in \mathcal{T}}$.

ProOF. Consider $\mathcal{H}$ composed of those elements

$$
\left(\left(\nu_{r}^{*}\right)_{r \in P}: P \subset \mathbb{N}, \nu_{r}^{*} \in \mathcal{M}_{r} \text { and } \pi_{r s}\left(\nu_{s}^{*}\right)=\nu_{r}^{*} \text { if } r, s \in P \text { and } r \leq s\right) .
$$

The latter lemma proves that $\mathcal{H}$ is non void. Consider the natural order of $\mathcal{H}$, i.e., a new element of $\mathcal{H}$ is greater than the element above if $P$ increases to $P^{\prime}$ and the previous measures $\nu_{r}^{*}$ remain constant for $r \in P$. It is easy to see that $\mathcal{H}$ is inductive, so the Zorn's Lemma ensures the existence of a maximal element. Suppose that (14) is this maximal. If $P$ is cofinal with $\mathbb{N}$ then it is easy to see that (14) is a projective system of Radon measures whose associated projective system of topological spaces has a projective limit that can be identified with $\left(\mathbb{R}^{n}\right)^{\mathcal{T}}$. Moreover, Theorem 2 ensures the existence of $\nu^{*}$, projective limit of $\left(\nu_{r}^{*}\right)_{r \in P}$, and it is easy to prove that $\nu^{*}$ satisfies the required conditions. In particular, $\operatorname{Sp}\left(\nu^{*}\right) \subset \prod_{t \in \mathcal{T}} K_{t}$ trivially follows from (10) and the projective equivalence between $\nu^{*}$ and $S_{\mathcal{T}}(\mu)$.

Suppose that $P$ is not cofinal. Then there exists its maximum value $r \in P$. Since $\nu_{r}^{*} \in \mathcal{M}_{r s}$ for ever $s \geq r$, set $\left(\nu_{s}^{*}\right)_{s=r}^{\infty}$ such that $\nu_{s}^{*} \in \mathcal{M}_{s s}$ and

$$
\nu_{r}^{*}=\pi_{r s}\left(\nu_{s}^{*}\right)
$$

Set

$$
\nu_{r+1, s}^{*}=\pi_{r+1, s}\left(\nu_{s}^{*}\right)
$$

for every $s>r$. Expression (15) shows that $\nu_{r}^{*}=\pi_{r, r+1}\left(\nu_{r+1, s}^{*}\right)$ for every $s \geq r$. If we were able to prove the existence of $\nu_{r+1}^{*}$, agglomeration point of $\left(\nu_{r+1, s}^{*}\right)_{s=r+1}^{\infty}$, we would have that

$$
\nu_{r}^{*}=\pi_{r, r+1}\left(\nu_{r+1}^{*}\right)
$$

Furthermore, being $\nu_{r+1}^{*}$ agglomeration point of $\left(\nu_{r+1, s}^{*}\right)_{s=r+1}^{\infty}$ and taking into account that (16) leads to

$$
\nu_{r+1, s}^{*} \in \mathcal{M}_{r+1, s} \subset \mathcal{M}_{r+1, r+1}
$$

for every $s \geq r+1$, one has that $\nu_{r+1}^{*} \in \mathcal{M}_{r+1}=\bigcap_{s=r+1}^{\infty} \mathcal{M}_{r+1, s}$ from where (17) generates a contradiction since (14) is not maximal. Thus $P$ must be cofinal.

It only remains to prove the existence of the agglomeration point $\nu_{r+1}^{*}$ but this is an obvious consequence of (18) and the weak ${ }^{*}$-compactness of $\mathcal{M}_{r+1, r+1}$.

It is worth to remark that minor modifications of the proof above can allow us to use a simple induction method rather than the Zorn's Lemma. Therefore, a more constructive proof of Theorem 5 may be available.

Notice that there are countable sets that are dense in the real line, which (under special assumptions such as continuous paths) might lead to new proofs of Theorem 5 that do not require the Zorn's Lemma, but strictly weaker axioms.

As said in the introduction this result extends those findings of Balbás et al. [5, (2002)], since there are no additional conditions on the countable set $\mathcal{T}$. Nevertheless there are many relationships between both analyses. So, in both cases the projective system approach has enlarged the set of states of the world so that the new set of states can contain the whole family of feasible paths of the price process (notice that the new set of states of nature is $\left(\mathbb{R}^{n}\right)^{\mathcal{T}}$ in the present case). As pointed out in Balbás et al. [5, (2002)], this fact implies that $\nu^{*}$ and $S_{\mathcal{T}}(\mu)$ cannot be equivalent since $\nu^{*}$ must vanish over the set of new states that can not be identified with any $\omega \in \Omega$. Consequently we have that the concept of projective equivalence is strictly weaker than the concept of equivalence (see Balbás et al. [5, (2002)] for further details). 


\section{Conclusions}

Representation Theorems have shown to be crucial in Mathematical Finance. Regarding pricing rules of perfect markets, for an infinite number of trading dates the characterization of the absence of arbitrage by the existence of equivalent martingale measures presents some difficulties, and the price process of the assets needs less intuitive notions such as "no free lunch" or "no free lunch with bounded risk", generalizing the concept of "no arbitrage". Moreover some constraints on the trajectories of the price process are usually required.

This paper considers a countable set of trading dates and draws on the projective system approach. Then we establish the equivalence between the absence of arbitrage and the existence of martingale measures. The equivalence holds under quite general assumptions since there are no conditions on the set of trading dates or on the trajectories of the price process.

The projective system approach allows us to enlarge the set of states of nature and to identify this set and the set of feasible trajectories. Thus a complete equivalence between the initial probability measure and the martingale measure does not hold in general. However the existence of densities between the "real" probabilities and the "risk-neutral" probabilities is guaranteed by introducing the concept of "projective equivalence", in the sense that both the martingale measure and the initial probability measure generate equivalent projections.

Acknowledgement. Research partially supported by "Comunidad Autónoma de Madrid" (Spain), Grant s-0505/tic/000230, and "MEyC" (Spain), Grant SEJ2006-15401-C04. The usual caveat applies.

\section{References}

[1] Artzner, P., Delbaen, F., Eber, J. M. and Heath, D., (1999). Coherent measures of risk, Math. Finance, 9, 3, 203-228.

[2] BaCK, K. AND Pliska, S. R., (1991). On the fundamental theorem of asset pricing with an infinite state space, J. Math. Econom., 20, 1-18.

[3] Balbás, A., BAlBÁs, R. And MAYORAL, S., (2007). Risk-neutral valuation with infinitely many trading dates, Mathematical \& Computer Modelling, 45, 11, 1308-1318.

[4] Balbás, A. And Downarowicz, A., (2007). Infinitely many securities and the fundamental theorem of asset pricing, Mediterr. J. Math., 4, 3, 321-341.

[5] Balbás, A., Mirás, M. And Muñoz-Bouzo, M. J., (2002). Projective system approach to the martingale characterization of the absence of arbitrage, J. Math. Econom., 37, 4, 311-323.

[6] Bourbaki, N., (1969). Éléments de mathématique Chapitre IX, Intégration, Diffusion C.C.L.S., Paris.

[7] Castagnoli, E., Maccheroni, F. And Marinacci, M., (2004). Choquet insurance pricing: A caveat, Math. Finance, 14, 3, 481-485.

[8] Chamberlain, G. And Rothschild, M., (1983). Arbitrage, factor structure, and mean-variance analysis on large assets, Econometrica, 51, 1281-1304.

[9] Chateauneuf, A., Kast, R. And Lapied, A., (1996). Choquet pricing for financial markets with frictions, Math. Finance, 6, 3, 323-330.

[10] Clark, S. A., (1993). The valuation problem in arbitrage price theory, J. Math. Econom., 22, 5, 463-478.

[11] Dalang, R. C., Morton, A. And Willinger, W., (1990). Equivalent martingale measures and no-arbitrage in stochastic securities market models, Stochastics and Stochastic Reports, 29, 185-201.

[12] De Waegenaere, A., Kast, R. And Lapied, A., (2003). Choquet pricing and equilibrium, Insurance Math. Econom., 32, 359-370. 
[13] Delbaen, F. And Schachermayer, W., (1998). The fundamental theorem of asset pricing for unbounded stochastic processes, Math. Ann., 312, 2, 215-250.

[14] Föllmer, H. And Schachermayer, W., (2008). Asymptotic arbitrage and large deviations, Math. Financ. Econ., 1, 3, 213-249.

[15] Hansen, L. P. and Jagannathan, R., (1997). Assessing specification errors in stochastic discount factor models, The Journal of Finance, 52, 2, 567-590.

[16] HARRISON, J. AND KREPS, D., (1979). Martingales and arbitrage in multiperiod securities markets, Journal of Economic Theory, 20, 381-408.

[17] JACOD, J. And Shiryaev, A., (1998). Local martingales and the fundamental asset pricing theorems in the discrete-time case, Finance Stoch., 2, 3, 259-273.

[18] Jouini, E. AND Kallal, H., (1995). Martingales and arbitrage in securities markets with transaction costs, Journal of Economic Theory, 66, 178-197.

[19] Kelley, J., (1955). General topology, D. Van Norstrand Company Ind. New York.

[20] Kopp, P. E., (1984). Martingales and stochastic integrals, Cambridge University Press, Cambridge.

[21] Musial, K., (1980). Projective limits of perfect measure spaces, Fund. Math., 40, 163-189.

[22] Schachermayer, W., (1992). A Hilbert space proof of the fundamental theorem of asset pricing in finite discrete time, Insurance Math. Econom., 11, 4, 249-257.

[23] Schachermayer, W., (1994). Martingale measures for discrete-time processes with infinite horizon, Math. Finance, 4, 1, 25-55.

[24] SchwartZ, L., (1973). Radon measures on arbitrary topological spaces and cylindrical measures, Oxford University Press. London.

\author{
Alejandro Balbás \\ Académico Correspondiente de la \\ Real Academia de Ciencias. \\ Universidad Carlos III de Madrid. \\ CL. Madrid, 126. \\ 28903 Getafe (Madrid, Spain). \\ alejandro.balbas@uc3m.es
}

\section{Pedro Jiménez Guerra}

Académico de Número de la

Real Academia de Ciencias.

UNED, Departamento de Matemáticas Fundamentales.

CL. Senda del Rey, 9.

28040 Madrid (Spain).

pjimenez@mat. uned.es 